\title{
The Rossby wave instability in protoplanetary disks
}

\author{
H. Meheut ${ }^{1,2, a}$ \\ 1 Physikalisches Institüt \& Center for Space and Habitability, Universität Bern, 3012 Bern, \\ Switzerland \\ 2 CEA, Irfu, SAp, Centre de Saclay, F-91191 Gif-sur-Yvette, France
}

\begin{abstract}
The Rossby wave instability has been proposed as a mechanism to transport angular momentum in the dead zone of protoplanetary disks and to form vortices. These vortices are of particular interest to concentrate solids in their centres and eventually to form planetesimals. Here we summarize some recent results concerning the growth and structure of this instability in radially and vertically stratified disks, its saturation and non-linear evolution. We also discuss the concentration of solids in the Rossby vortices including vertical settling.
\end{abstract}

\section{Introduction}

The formation of planetesimals of kilometre size is still an issue due to the rapid drift of solids grains towards the central star and the collisions that can lead to their destruction (Youdin \& Kenyon, 2012). A promising scenario involves the existence of anticyclonic vortices that are able to concentrate a large amount of dust and grains in their centres and to accelerate the growth process (Barge \& Sommeria, 1995; Chavanis, 2000). A possible path to form such vortices is the Rossby wave instability (RWI). This instability, initially studied by Lovelace et al. (1999) (see also Li et al. 2000; Umurhan 2010), has some similarities with the Papaloizou-Pringle instability (Papaloizou \& Pringle, 1985). Its growth mechanism is attributable to the interaction of two Rossby waves of respectively positive and negative energy. Rossby waves are vorticity waves propagating in a gradient of specific vorticity and they are responsible for the formation of the vortices. Thus, this instability needs the presence of an extremum of specific vorticity to grow. Such an extremum can be due to a density bump forming at the interface between active and dead zones (Varnière \& Tagger, 2006; Lyra \& Mac Low, 2012) or at the boundaries of planet gaps (Lin \& Papaloizou, 2011). Differential rotation couples these Rossby waves with density waves in the regions exterior of their forbidden zone, namely outside the Lindblad resonances. These density waves carry away energy and angular momentum from the bump region.

We will present in the next section some properties of the linear instability in a 3D disk and its saturation in section 3. Their ability to concentrate solids is discussed in section 4 and the long-term evolution of the vortices in section 5. We then summarize the results presented here.

\section{Linear growth in 3D}

We have studied the linear growth of the Rossby wave instability (Meheut et al., 2012d) by solving the linearized Euler equations for a perfect fluid in the gravitational field of the star. Here the disk is considered fully isothermal. The azimuthal and vertical components are decomposed on a Fourrier and Hermite basis. This linear analysis showed that the growth rate obtained in 3D with vertical stratification is only slightly lower than the growth rate computed in a razor thin disk. The eigenfunctions for

\footnotetext{
a e-mail: heloise.meheut@cea.fr
}

This is an Open Access article distributed under the terms of the Creative Commons Attribution License 2.0, which permits unrestricted use, distribution, and reproduction in any medium, provided the original work is properly cited. 

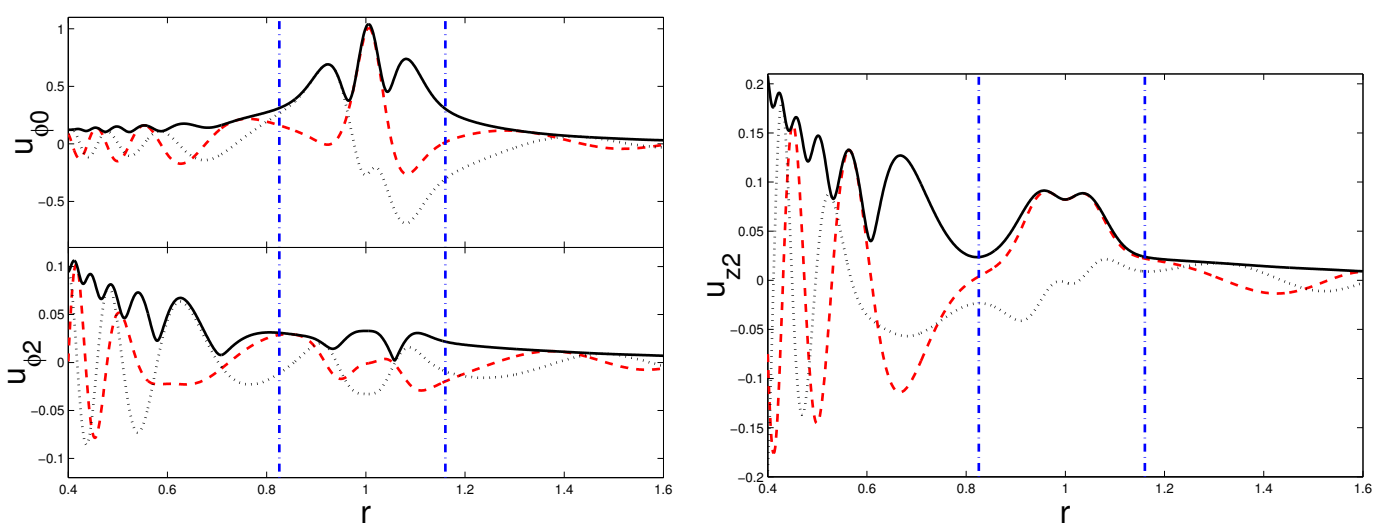

Fig. 1. Radial profile of the azimuthal (left) and vertical velocity obtained with the linear analysis. For the azimuthal velocity, the two components of the Hermite transform are plotted. The dashed line is the real part of the Fourier transform, the dotted line the imaginary part and the solid line is the amplitude.

the azimuthal and vertical velocities are shown on Fig.1. The azimuthal velocity has two components, the vertically constant $\left(u_{\phi 0}\right)$ and linearly growing $\left(u_{\phi 2}\right)$ parts. The vertical velocity has a small but non-zero amplitude which could not be captured in previous $2 \mathrm{D}$ analysis. This vertical velocity can be of importance for the study of the dust concentration in the vortices.

A similar approach has been used by Lin (2012b) (see also Lin (2012a)) but with different disk thermodynamics. It showed that the amplitude of the vertical velocity varies with the disk thermodynamics and can vanish.

\section{Non-linear simulations and saturation}

We have performed global 3D non-linear numerical simulations of the RWI and we obtained similar results than in the linear analysis. The growth rate are compatible and the same vertical velocity has been obtained. In the simulations of Meheut et al. (2010, 2012a) where a polytropic disk was considered, a large scale vertical velocity has also been obtained in the linear phase of the instability. The non-linear simulations also allowed to investigate the saturation mechanism of the instability. Lovelace et al. (2009) have proposed that the instability should saturate when the turnover time in the vortices reaches the instability growth timescale. This hypothesis has been tested against the simulations (Meheut et al., 2012b) and the amplitudes of vorticity at saturation do correspond with the model. For instance, the left part of Fig.2 shows the growth of the instability and a fit of the growth rate in the non-linear simulation. The dashed line corresponds to the saturation model. The ratio of the expected vorticity at saturation and the numerical result is 1.01 for this simulation. On the other hand for higher azimuthal mode number, when the density waves are of higher amplitude and become non-linear, they take away energy from the corotation region and the maximum amplitude at saturation is lowered (Fig. 2 , right).

\section{Concentration of solids in the vortices}

One specificity of the RWI is the formation of vortices that can concentrate solids. This mechanism can be altered by the vertical velocity in the central part of the vortices. For this reason, the concentration of solids in the 3D Rossby vortices has been studied numerically (Meheut et al., 2012c). Here solids are included in the simulation after the formation of the vortices and the co-evolution of solids and gas is followed over a few orbits. Note that the back-reaction of the solids on the gas is included. The larger solids (e.g. $2 \mathrm{~cm}$ ) are rapidly concentrated and settled into the core of the vortices. Whereas the initial 

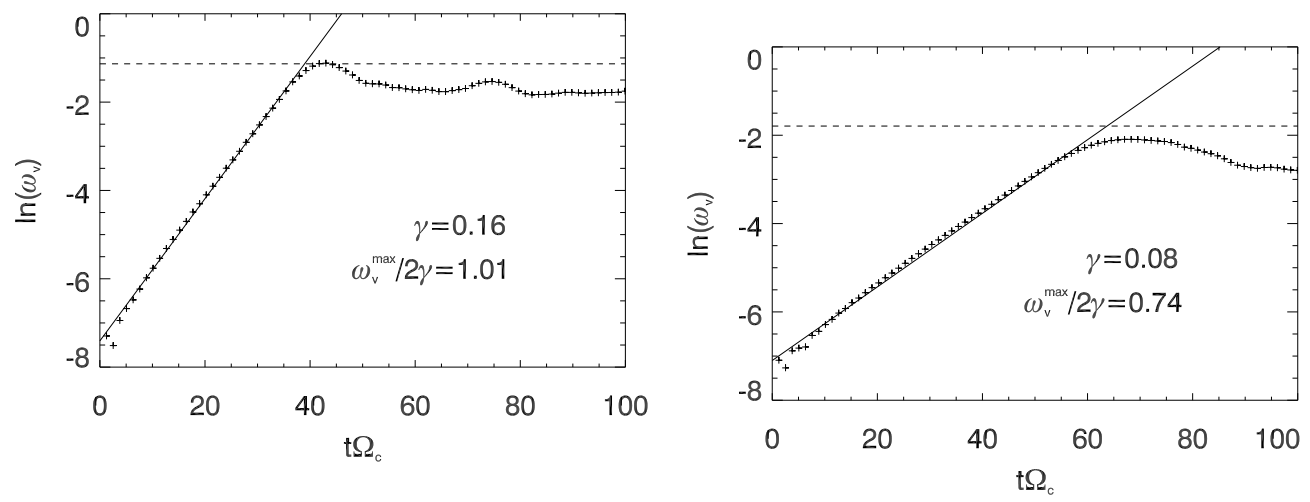

Fig. 2. Growth of the vorticity in the non-linear simulations, for two different azimuthal mode number: $m=4$ (left) and $m=5$ (right). The solid line is a fit of the numerical datas to estimate the growth rate, the dashed line is the predicted amplitude of saturation.

dust-to-gas density ratio is $10^{-2}$, the solids density reaches the gas density in a few orbits. The vertical density profile at the radius of the vortices is plotted on the upper part of Fig.3. The intermediate size solids $(5 \mathrm{~mm})$ have an interesting behavior. They are concentrated into the vortices but the highest solid density is reached above the midplane at the upper limit of the disk of solids. This surprising result is due to the gas vertical velocity inside the vortices that is responsible for the lifting of the solid grains. This can be seen on the lower part of Fig.3. An other interesting results is the modification of the structure of the vortices when the solid density reaches the amplitude of the the gas density and the solids start to drag the gas.

\section{Long-term evolution}

The long-term stability of the 3D vortices has often been questioned (e.g. Barranco \& Marcus 2005; Lesur \& Papaloizou 2009) due to parasitic instabilities. This long-term stability over a few hundreds years has been investigated in non-linear simulations (Meheut et al., 2012a). Whereas the RWI tends to form several vortices, after the saturation of the instability, they tend to merge to form only one larger vortex. The vortices enhanced by the RWI are stable due to their particular 3D structure with no close streamlines, their structure is modified and they start to decay when the RWI stops to be active. Simulations with a higher resolution to capture the elliptical instability (Lesur \& Papaloizou, 2009) and eventually in a baroclinic disk to capture the baroclinic instability (Klahr \& Bodenheimer, 2003) should be performed to have a better understanding of this long-term evolution. Moreover, the simulations should also include the continuous process responsible for the formation of the density bump. If the bump continues to be enhanced, we do not expect the RWI to be suppressed.

\section{Summary}

If a mechanism can form an extremum of specific vorticity in protoplanetary disks, the RWI can linearly grow. The vertical stratification does not strongly modify the instability but it can induce a vertical component in the structure of the flow depending on its thermodynamics. After a few orbits the instability saturates. This saturation occurs when the turnover timescale inside the vortices is of the order magnitude of the instability growth timescale. The study of the evolution of solids particules in those vortices has shown that the solid density can be increased by a factor 100 in the centres of the vortices on a few dynamical timescales. Such high concentration should be profitable for the formation of planetesimals. On longer timescale, the RWI stops to be active due to the decay of the initial bump. 


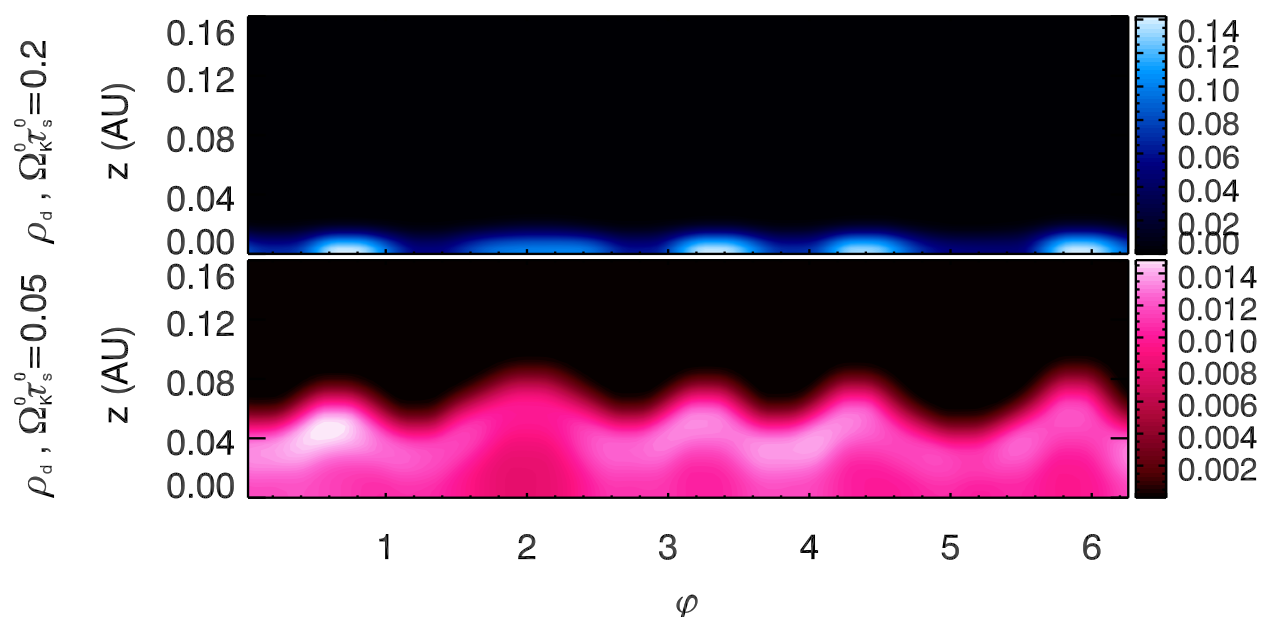

Fig. 3. Vertical density profile of the $2 \mathrm{~cm}$ and $5 \mathrm{~mm}$ dust population at the radius of the 5 vortices. The larger grains have settled whereas the $5 \mathrm{~mm}$ grains are lifted by the gas and have a higher density in the upper part of the dust disk.

The structure of the vortices then evolves and they loose their fully 3D structure and tend to be more elliptical. In the configuration chosen here, they eventually decay after a few hundred years.

\section{References}

Barge, P., \& Sommeria, J. 1995, A\&A , 295, L1

Barranco, J. A., \& Marcus, P. S. 2005, ApJ , 623, 1157

Chavanis, P. H., P. H. 2000, A\&A, 356, 1089

Klahr, H. H., \& Bodenheimer, P. 2003, ApJ , 582, 869

Lesur, G., \& Papaloizou, J. C. B. 2009, A\&A, 498, 1

Li, H., Finn, J. M., Lovelace, R. V. E., \& Colgate, S. A. 2000, ApJ , 533, 1023

Lin, M.-K. 2012a, ArXiv e-prints

-. 2012b, ApJ , 754, 21

Lin, M.-K., \& Papaloizou, J. C. B. 2011, MNRAS , 415, 1445

Lovelace, R. V. E., Li, H., Colgate, S. A., \& Nelson, A. F. 1999, ApJ , 513, 805

Lovelace, R. V. E., Turner, L., \& Romanova, M. M. 2009, ApJ , 701, 225

Lyra, W., \& Mac Low, M.-M. 2012, ApJ , 756, 62

Meheut, H., Casse, F., Varniere, P., \& Tagger, M. 2010, A\&A , 516, A31+

Meheut, H., Keppens, R., Casse, F., \& Benz, W. 2012a, A\&A, 542, A9

Meheut, H., Lovelace, R. V. E., \& Lai, D. 2012b, MNRAS , subm.

Meheut, H., Meliani, Z., Varniere, P., \& Benz, W. 2012c, A\&A, 545, A134

Meheut, H., Yu, C., \& Lai, D. 2012d, MNRAS , 2748

Papaloizou, J. C. B., \& Pringle, J. E. 1985, MNRAS , 213, 799

Umurhan, O. M. 2010, A\&A , 521, A25+

Varnière, P., \& Tagger, M. 2006, A\&A , 446, L13

Youdin, A. N., \& Kenyon, S. J. 2012, ArXiv e-prints 